\title{
Effects of pentosan polysulfate and polysulfated glycosaminoglycan on chondrogenesis of canine bone marrow-derived mesenchymal stem cells in alginate and micromass culture
}

J. Vet. Med. Sci.

79(7): 1182-1190, 2017

doi: 10.1292/jvms.17-0084

Received: 20 February 2017

Accepted: 15 May 2017

Published online in J-STAGE:

27 May 2017

\author{
Eugene C. BWALYA ${ }^{1) *}$, Sangho KIM ${ }^{1}$, Jing FANG ${ }^{1}$, H. M. Suranji WIJEKOON ${ }^{1}$, \\ Kenji HOSOYA ${ }^{1)}$ and Masahiro OKUMURA ${ }^{1)}$
}

1)Laboratory of Veterinary Surgery, Department of Veterinary Clinical Sciences, Graduate School of Veterinary Medicine, Hokkaido University, Sapporo, Hokkaido 060-0818, Japan

\begin{abstract}
Mesenchymal stem cells (MSC) are a potential alternative source of differentiated chondrocytes for cartilage tissue regeneration and repair of osteoarthritic (OA) joints. We investigated the effects of pentosan polysulfate (PPS) and polysulfated glycosaminoglycan (PSGAG) on chondrogenesis of canine bone marrow-derived mesenchymal stem cells (cBMSC) in alginate and micromass cultures (MMC). Chondrogenic differentiation medium (CDM) was supplemented with PPS or PSGAG at concentrations of 0 (positive control; PC), 1,3 and $5 \mu \mathrm{g} / \mathrm{m} /$. $10 \%$ DMEM was used as negative control. Chondrocyte phenotype was analyzed by quantitative real-time PCR (qPCR) for alginate cultures and Alcian blue staining for proteoglycan (PG) synthesis for MMC. In alginate culture, PPS and PSGAG showed no significant effect on type Il collagen, aggrecan and HIF-2a mRNA expression. PPS had no significant effect on type I collagen whereas PSGAG significantly upregulated $(P<0.05)$ it at all concentrations relative to other treatments. PPS demonstrated a dose-dependent inhibitory effect on type $X$ collagen mRNA with significant inhibition observed at $5 \mu \mathrm{g} / \mathrm{m} /$ compared to the NC. PSGAG showed an inverse effect on type $X$ collagen with $1 \mu \mathrm{g} / \mathrm{m} /$ significantly inhibiting its expression while increase in the concentration correspondingly increased type $X$ collagen expression. In MMC, PPS significantly enhanced chondrogenesis and PG deposition whereas PSGAG inhibited chondrogenesis and promoted a fibrocartilage-like phenotype with reduced PG deposition. While PPS enhances chondrogenesis of CBMSC in MMC, the response of MSC to chondroinductive factors is culture system-dependent and varies significantly between alginate and MMC.
\end{abstract}

KEY WORDS: canine bone marrow-derived mesenchymal stem cells, cartilage tissue regeneration, osteoarthritis, pentosan polysulfate, polysulfated glycosaminoglycan 
to differentiate into a variant of cells $[5,15]$. These undifferentiated cells can differentiate into different generative cells such as bone, cartilage, adipose, tendon and other cells of the mesenchymal lineage under appropriate stimuli. Therefore, MSC are a potential alternative source of differentiated chondrocytes from different sources including bone marrow-derived mesenchymal precursor cells (MPC) [15] for cartilage tissue regeneration and repair of OA joints. Previous studies have shown that MSC just like monolayer cultured articular chondrocytes concomitantly express the undesirable dedifferentiation and hypertrophic markers, thus raising questions on their suitability for cartilage tissue regenerative.

Therefore, these challenges at the front-end of cartilage tissue regeneration demands that novel in vitro culture conditions are developed to engineer a cell population with a stable chondrocyte phenotype. In the present study we explored the effects of pentosan polysulfate (PPS) and polysulfated glycosaminoglycan (PSGAG) on chondrogenic differentiation of MSC with a view of improving the culture conditions for cartilage tissue engineering. Pentosan polysulfate (PPS) a semi-synthetic sulfated polysaccharide derived from wood of beech plant (Fagus sylvatica) has been shown to improve synovial and subchondral blood flow, stimulate hyaluronic acid (HA) and proteoglycan (PG) synthesis, limit cartilage matrix degeneration and suppress catabolic matrix metalloproteinases $(M M P S)$ [7, 9, 10, 14, 20, 30, 34]. Our laboratory recently showed its involvement in the prevention of inflammatory intracellular responses induced by interleukin 1-beta $(I L-1 \beta)$ through inhibition of phosphorylation of certain $M A P K s$, p38 and ERK [40]. Of particular interest is a recent study finding which showed that PPS promotes human MPC proliferation and chondrogenesis in pellet or micromass culture (MMC) [15]. Similar to PPS, polysulfated glycosaminoglycan (PSGAG) a semisynthetic polysulfated chondroitin sulfate has been shown to diminish articular cartilage matrix molecule degradation [13, 35, 43], improve lameness score [13] and enhance matrix molecule synthesis [16]. However, there are no studies that have evaluated its effects on chondrogenesis of MSC.

Generally, the use of bioscaffold 3-D culture systems is increasingly being employed to better mimic the in vivo chondrocyte environment. The use of alginate culture provides a natural material for 3-D encapsulation of cells which has been shown to support chondrogenesis and redifferentiation of chondrocytes $[4,12,26,37]$. Therefore, the objective of the study was to investigate the effects of PPS and PSGAG on chondrogenesis of canine bone marrow-derived MSC (cBMSC) in alginate culture and MMC.

\section{MATERIALS AND METHODS}

\section{Isolation, culture and differentiation of $c B M S C$}

Collection site and dog preparation: The study was performed using cBMSC aseptically collected by the proximal femur approach from femoral bone marrow of three experimental Beagle dogs (age range; 11 to 12 months old). The use of experimental dogs was in accordance with Hokkaido University Institutional Animal Care and Use Committee guidelines (approval number: 120059). Briefly, dogs were put under general anesthesia (GA) using propofol at $6 \mathrm{mg} / \mathrm{kg}$ intravenously for induction and maintained on isoflurane and oxygen. The limb from where the bone marrow aspirate was collected was aseptically prepared by clipping the hair around the proposed site of collection, scrubbed with povidone iodine and then $70 \%$ ethanol applied to further disinfect the site.

cBMSC isolation and culture: Isolation and culture of cBMSC was performed using the protocol previously described by Soleimani and Nadri (2009) [38] with slight modification which has been validated to obtain a purified population of MSC with mesenchymal lineages differentiation ability. Briefly, mononuclear cells (MC) were collected by gradient centrifugation using a modification of Lymhoprep ${ }^{\text {TM }}$ protocol $(1.077 \pm 0.001 \mathrm{~g} / \mathrm{ml}$ ) (Axis Shield POC, Oslo, Norway). Briefly, after centrifugation, $\mathrm{MC}$ were collected from the sample/medium interface and plated in polystyrene culture plates containing Dulbecco's Modified Eagle Medium (DMEM) (GIBCO BRL, Grand Island, NY, U.S.A.) supplemented with 10\% fetal bovine serum (FBS) (Nichirei Biosciences Inc., Tokyo, Japan, Batch \#: 83300104), $100 \mathrm{IU} / \mathrm{m} l$ of penicillin and $0.1 \mathrm{mg} / \mathrm{m} l$ of streptomycin and incubated at $37^{\circ} \mathrm{C}$ in $5 \% \mathrm{CO}_{2}$ in a humidified chamber without disturbing the plates for 4 days. Thereafter, non-adherent cells were removed, cells gently washed with $1 \times$ PBS and fresh medium added. Medium was changed every $48 \mathrm{hr}$ until the cells reached about 80-85\% confluence. At confluence, primary (P0) monolayer colony forming unit cells were gently washed twice with $1 \times$ PBS and detached using pre-warmed TrypLE ${ }^{\mathrm{TM}}$ Select $\mathrm{CTS}^{\mathrm{TM}}$ (GIBCO). The isolated cells exhibited stemness character based on their ability to adhere to plastic culture plate surface, morphology, non-expression of CD34 and CD45, and ability to differentiate upon specific induction into chondrogenic, adipogenic and osteogenic lineage as verified by Alcian blue, Oil red O and Alizarin red S staining, respectively. Cell viability and total cell density was determined using Trypan blue stain and manual hemocytometer cell counting method. P0 were passaged and expanded twice with subsequent P1 and P2 cells being differentiated into chondrogenic lineage using complete chondrogenic differentiation medium (CDM) (StemPro ${ }^{\circledR}$ Chondrogenesis Differentiation Kit, GIBCO BRL) exogenously supplemented with either PPS (Cartrophen Vet ${ }^{\circledR}$ injection NaPPS-100 mg/ml; Biopharm Australia, NSW, Australia) or PSGAG (Adequan ${ }^{\circledR}$ PSGAG-100 mg/ml; Novartis Animal Health Inc., Tokyo, Japan) at concentrations of 1, 3 and $5 \mu \mathrm{g} / \mathrm{m} l$. The negative control (NC) cells were cultured in 10\% DMEM while the positive control (PC) group was cultured in CDM. P1 and P2 cells derived from each dog were encapsulated and cultured independently in the presence or absence of PPS and PSGAG, giving a total of six (6) independent experiments for each chondrogenesis assay.

Alginate beads culture: The differentiation of cBMSC using alginate beads culture was based on the protocol described elsewhere [12], which has the potential to differentiate MSC into chondrocytes [8]. Briefly, cells were suspended in $1 \mathrm{~m} l$ of $1.25 \%$ (w/v) sodium alginate (Wako Pure Chemical Industries Ltd., Osaka, Japan, Product code: 192-09951) solution. The suspension was diluted progressively until a homogenous cellular density of $2 \times 10^{6}$ cells $/ \mathrm{m} l$ was reached. The cell suspension was expressed in drops through a 22-gauge needle into the gelation solution of $102 \mathrm{mM}$ calcium chloride in six-well plates (Corning) and allowed to polymerize for $15 \mathrm{~min}$ to form beads. The beads were washed three (3) times with $0.15 \mathrm{M} \mathrm{NaCL}$ then once with $10 \%$ DMEM 
Table 1. Sequence of primers used for reverse transcriptase PCR and real-time PCR to evaluate the effects of pentosan polysulfate and polysulfated glycosaminoglycan on gene expression in canine bone marrow-derived mesenchymal stem cells cultured in alginate beads and micromass

\begin{tabular}{|c|c|c|c|c|}
\hline Name of gene & Domain & Primer $^{a)}$ & Fragment $^{\mathrm{b})}(\mathrm{bp})$ & Accession \\
\hline \multirow[t]{2}{*}{ GAPDH } & $664-683$ & 5'-CTGAACGGGAAGCTCACTGG-3' & 129 & NM_001003142.1 \\
\hline & $773-792$ & 5'-CGATGCCTGCTTCACTACCT-3' & & \\
\hline \multirow[t]{2}{*}{ Sox-9 } & $565-583$ & 5 '-GCCGAGGAGGCCACCGAACA-3' & 179 & NM_001002978.1 \\
\hline & $724-743$ & 5'-CCCGGCTGCACGTCGGTTTT-3' & & \\
\hline \multirow[t]{2}{*}{ Type I Collagen } & $150-169$ & 5'-GTGGATACGCGGACTTTGTT-3' & 164 & NM_001003187.1 \\
\hline & $294-313$ & $5^{\prime}$-GGGATACCATCGTCACCATC-3' & & \\
\hline \multirow[t]{2}{*}{ Type II Collagen } & $4127-4146$ & 5'-CACTGCCAACGTCCAGATGA-3' & 215 & NM_001006951.1 \\
\hline & $4322-4341$ & 5'-GTTTCGTGCAGCCATCCTTC-3' & & \\
\hline \multirow[t]{2}{*}{ Type X Collagen } & $1782-1801$ & 5'-TTTCTCCTACCACGTGCATG-3' & 117 & XM_003639401.1 \\
\hline & $1879-1898$ & 5'-GAAGCCTGATCCAGGTAGCC-3' & & \\
\hline \multirow[t]{2}{*}{ Aggrecan } & $6569-6588$ & 5'-ACTTCCGCTGGTCAGATGGA-3' & 111 & NM_001113455.1 \\
\hline & $6660-6679$ & 5'-TCTCGTGCCAGATCATCACC-3' & & \\
\hline \multirow[t]{2}{*}{ HIF- $2 \alpha$} & $1248-1267$ & 5'-TGCAAAGCACGGGGGCTACG-3' & 72 & XM_531807.3 \\
\hline & $1300-1319$ & 5'-GGCTGCAGGTTGCGAGGGTT-3' & & \\
\hline
\end{tabular}

a) Primers for forward \& reverse sense are presented in a 5' to 3' orientation, b) The expected fragment size, GAPDH=glyceraldehyde-3-phosphate dehydrogenase, HIF-=Hypoxia inducible factor-.

before being assigned specific treatment and incubated at $37^{\circ} \mathrm{C}$ in a humidified atmosphere of $5 \% \mathrm{CO}_{2}$. Alginate beads per culturewell contained an equivalent of $1 \times 10^{6}$ cells. The cultures were maintained for 20-days with medium changes done every $72 \mathrm{hr}$. On day-20, cells were recovered from alginate beads according to the protocol described elsewhere [12] and total RNA was extracted using TRIZol ${ }^{\circledR}$ Kit (Invitrogen, Life Technologies, Carlsbad, CA, U.S.A.), according to the manufacturer's instructions.

Micromass culture $(M M C)$ : Micromass cultures (MMC) were established according to StemPro® Chondrogenesis Differentiation Kit (GIBCO BRL). Briefly, cells were suspended in serum-free (SF) DMEM to generate a cell solution of $1.6 \times 10^{7} \mathrm{cells} / \mathrm{m} l$. MMC were established by seeding $20-\mu \mathrm{l}$ droplets of cell solution in the centre of 12 -well plate. The plates were incubated for $3 \mathrm{hr}$ to allow cell adherence then 10\% DMEM was gently added to each well and plates incubated overnight. After $24 \mathrm{hr}$, the medium was gently removed and each well was assigned specific treatment and incubated at $37^{\circ} \mathrm{C}$ in $5 \% \mathrm{CO}_{2}$. Medium was replenished every $72 \mathrm{hr}$. Chondrocyte phenotype was assessed by Alcian blue stain analysis for proteoglycan (PG) deposition at 12- and 20-days culture period.

PPS and PSGAG effects on chondrogenic differentiation of cBMSC: Chondrocyte phenotype analysis by real-time quantitative $P C R$ ( $q P C R$ ): Total RNA was quantified by spectrophotometry at $260 \mathrm{~nm}$. Total of $500 \mathrm{ng}$ RNA was reverse transcribed into cDNA using M-MLV RT kit (Invitrogen) according to manufacturer's recommended protocol. Quantitative real-time PCR (qPCR) was performed with KAPA SYBR ${ }^{\circledR}$ FAST qPCR kit (KAPA biosystems, Woburn, MA, U.S.A.) to determine the relative mRNA expression of the selected gene markers by the two step method; Sox-9, type II collagen and aggrecan as chondrocyte-specific genes, type I and X collagen as chondrocyte dedifferentiation and hypertrophy genes respectively, and hypoxia inducible factor- $2 \alpha$ $(H I F-2 \alpha)$. cDNA template with the amount of $2 \mu l$ was added to each $16 \mu l$ of kapa mix and $2 \mu l$ specific primers premixture. qPCR conditions were an initial denaturation of $95^{\circ} \mathrm{C}$ for $20 \mathrm{sec}$ followed by 40 cycles of $95^{\circ} \mathrm{C}$ for $3 \mathrm{sec}$ and $60^{\circ} \mathrm{C}$ for $20 \mathrm{sec}$ then a pre-melt condition of $60^{\circ} \mathrm{C}$ for $90 \mathrm{sec}$ followed by a final melt step. All PCR reactions were validated by the presence of a single peak in the melt curve analysis. The standard curve method was used to determine the relative mRNA quantification of selected genes between the treatments with the NC group as the calibrator. All target genes expression were normalized against the reference gene, glyceraldehyde-3-phosphate dehydrogenase $(G A P D H)$ making the variations in the number of cells per sample insignificant [11]. The sequences of primers used in the study are indicated in Table 1.

Chondrocyte phenotype analysis by Alcian blue stain: After 12- and 20-days of culture MMC pellets were evaluated for PG deposition by Alcian blue stain analysis. Briefly, at 12- and 20-day culture, the medium was removed from culture plates, pellets were gently washed once with $1 \times \mathrm{PBS}$ and then fixed in $4 \%$ formaldehyde solution for $30 \mathrm{~min}$. After fixation, the pellets were rinsed with $1 \times \mathrm{PBS}$ and stained with $1 \%$ Alcian blue solution prepared in $0.1 \mathrm{~N}$ HCL for 30 min. The pellets were rinsed three times with $0.1 \mathrm{~N} \mathrm{HCl}$, before adding distilled water to neutralize the acidity. The pellets were visualized under light microscope and images captured for PG analysis. Blue staining of the pellets indicate synthesis of cartilage sulfated aggrecan, a marker of chondrocyte phenotype.

\section{Data handling, storage and analysis}

Quantitative data was entered into a Microsoft Excel ${ }^{\circledR}$ spreadsheet. The data was then transferred to SPSS version 16.0 for descriptive and analytical statistics. Analysis of variance (ANOVA) was used to determine significant difference in the mRNA expression between the treatments. Where significant difference was observed, Post hoc multiple comparisons was performed using Bonferroni test to determine significantly different treatments. Unless specified, data is summarized as mean $\pm 95 \%$ confidence interval $(95 \% \mathrm{CI})$. Significant difference was defined as $P<0.05$. 


\section{RESULTS}

\section{Chondrogenic phenotype profile of alginate beads cultured cBMSC-qPCR}

Overall, the mean type I collagen mRNA expression tended to decrease with increase in PPS concentration $(1$ to $5 \mu \mathrm{g} / \mathrm{m} l)$. However, there was no significant difference $(P>0.05)$ between PPS treated beads when compared to NC and PC groups (Fig. 1). In contrast, PSGAG significantly upregulated $(P<0.05)$ type I collagen mRNA at all concentrations compared to NC, PC and PPS treated beads (Fig. 1). Both PPS and PSGAG had no significant effect on type II collagen, aggrecan and HIF-2 $\alpha$ mRNA expression although at $3 \mu \mathrm{g} / \mathrm{m} l$ both drugs tended to increase the expression of the three genes. PPS showed a dose-dependent inhibitory effect on type X collagen with the hypertrophy gene significantly inhibited at $5 \mu \mathrm{g} / \mathrm{m} l$ compared to the NC. Interestingly, PSGAG demonstrated an inverse inhibitory effect on type X collagen with a lower concentration of $1 \mu \mathrm{g} / \mathrm{m} l$ significantly inhibiting the hypertrophy marker compared to the $\mathrm{NC}$ whereas increase in concentration from 1 to $5 \mu \mathrm{g} / \mathrm{m} l$ correspondingly increased the gene expression (Fig. 1). The most provocative finding of the results was the undetectable Sox-9 mRNA. Nonetheless, cBMSC still exhibited a chondrocyte phenotype expressing cartilage-specific genes; type II collagen and aggrecan. This unusual finding by qPCR analysis was further verified with RT-PCR ethidium bromide gel electrophoresis which showed no Sox-9 mRNA bands in all the alginate cultured cBMSC albeit expressing type II collagen and aggrecan (Fig. 2A). To clarify whether the non-detection of Sox-9 mRNA was related to alginate culture, P1 cBMSC were cultured under monolayer condition for 20-days in CDM. Sox-9 mRNA was highly induced in monolayer culture suggesting that alginate may have suppressive effects on Sox-9 mRNA (Fig. 2B).

\section{PPS and PSGAG effects on PG deposition in MMC}

The effect of PPS and PSGAG on PG deposition in 12- and 20-days MMC was assessed by Alcian blue stain analysis. In 12days cultures in the NC group most cells predominantly proliferated as monolayer fibroblastic-like cells with limited chondrogenic condensation. Chondrogenic condensation of cBMSC was more pronounced in PC compared to the NC although PG deposition was not different (Fig. 3A and 3B). When compared to the NC and PC, both PPS and PSGAG tended to enhance chondrogenic pelleting and PG deposition in a dose-dependent pattern (Fig. 3C-3H). In 20-days culture, cBMSC cultured in 10\% DMEM predominantly proliferated as fibroblastic-like monolayer cells with limited chondrogenic pellets that stained poorly with Alcian blue indicating reduced PG deposition and chondrogenic differentiation (Fig. 4A). While cells cultured in CDM continued to form more compact chondrogenic pellets, some pellets stained poorly with Alcian blue stain. Interestingly, PPS demonstrated significantly enhanced chondrogenic pelleting and PG deposition at all concentrations when compared to the NC, PC and PSGAG, an indication of its potential to promote chondrogenic differentiation of cBMSC (Fig. 4C-4E). In contrast, in 20 days culture, PSGAG inhibited chondrogenic pelleting and PG deposition in a dose-dependent pattern with most pellets being characterized by less condensed and sparsely arranged fibroblastic-like cellular aggregates which stained poorly with Alcian blue, an indication of its potential to inhibit chondrogenic differentiation of cBMSC (Fig. 4F-4H).

\section{DISCUSSION}

The objective of our study was to investigate the effects of PPS and PSGAG on chondrogenic differentiation of cBMSC cultured in alginate beads and MMC with a view of improving in vitro chondrogenesis culture conditions for cartilage tissue regeneration. We hypothesized that PPS and PSGAG would synergistically enhance chondrogenic differentiation of cBMSC while concomitantly suppressing hypertrophy and dedifferentiation. The present study demonstrates that the response of cBMSC to chondroinductive factors is culture system-dependent and varies significantly between alginate culture and MMC. The most provocative finding of the study is the observed suppressive effect of alginate on transcription factor Sox-9 mRNA, a known positive regulator of articular cartilage differentiation. Nonetheless, cBMSC cultured in alginate beads still demonstrated a chondrocyte phenotype expressing type II collagen and aggrecan with no significant difference in the genes expression between PPS and PSGAG treated beads, and cells cultured in standard medium or only CDM. PPS had no significant effect on type I collagen expression while PSGAG significantly upregulated the gene at all concentrations. PPS inhibited type X collagen in a dose-dependent pattern with the highest concentration significantly inhibiting the hypertrophy gene compared to the NC. The observed inhibitory effects of PPS on type $X$ collagen are in agreement with findings of a previous study [15]. In contrast, PSGAG had an inverse effect on the hypertrophy gene with the lowest concentration significantly inhibiting the gene while increase in concentration correspondingly increased type $X$ collagen mRNA expression. We also demonstrate the extensive expression of $H I F-2 \alpha$ mRNA in alginate beads cultured cBMSC with both PPS and PSGAG having no significant effect on its expression when compared to the NC and PC. However, in MMC, PPS significantly enhanced chondrogenic condensation and PG deposition, a confirmation of its potential to promote chondrogenic differentiation. In contrast, we demonstrate for the first time that PSGAG has inhibitory effects on chondrogenic condensation and PG deposition in cBMSC, an indication of its potential to suppress chondrogenic differentiation.

The transcription factor Sox-9, is a known positive regulator of articular cartilage differentiation, chondrocyte proliferation, and transition to a non-mitotic hypertrophic state. It is highly activated and predominantly expressed in mesenchymal condensations before and during the deposition of cartilage [36, 39] and is necessary for chondrogenesis both before and after mesenchymal condensations [19, 27]. It activates specific enhancer elements in cartilage matrix genes and stimulates transcription of cartilage matrix genes, type II collagen and aggrecan [19, 23, 39]. While it has been reported that alginate supports chondrogenic differentiation of MSC, our findings strongly suggests that the encapsulation of cBMSC in alginate may have inhibitory effect on the process. This unusual finding is contrary to a previous study which demonstrated an increase in Sox- 9 with BMP treated human 

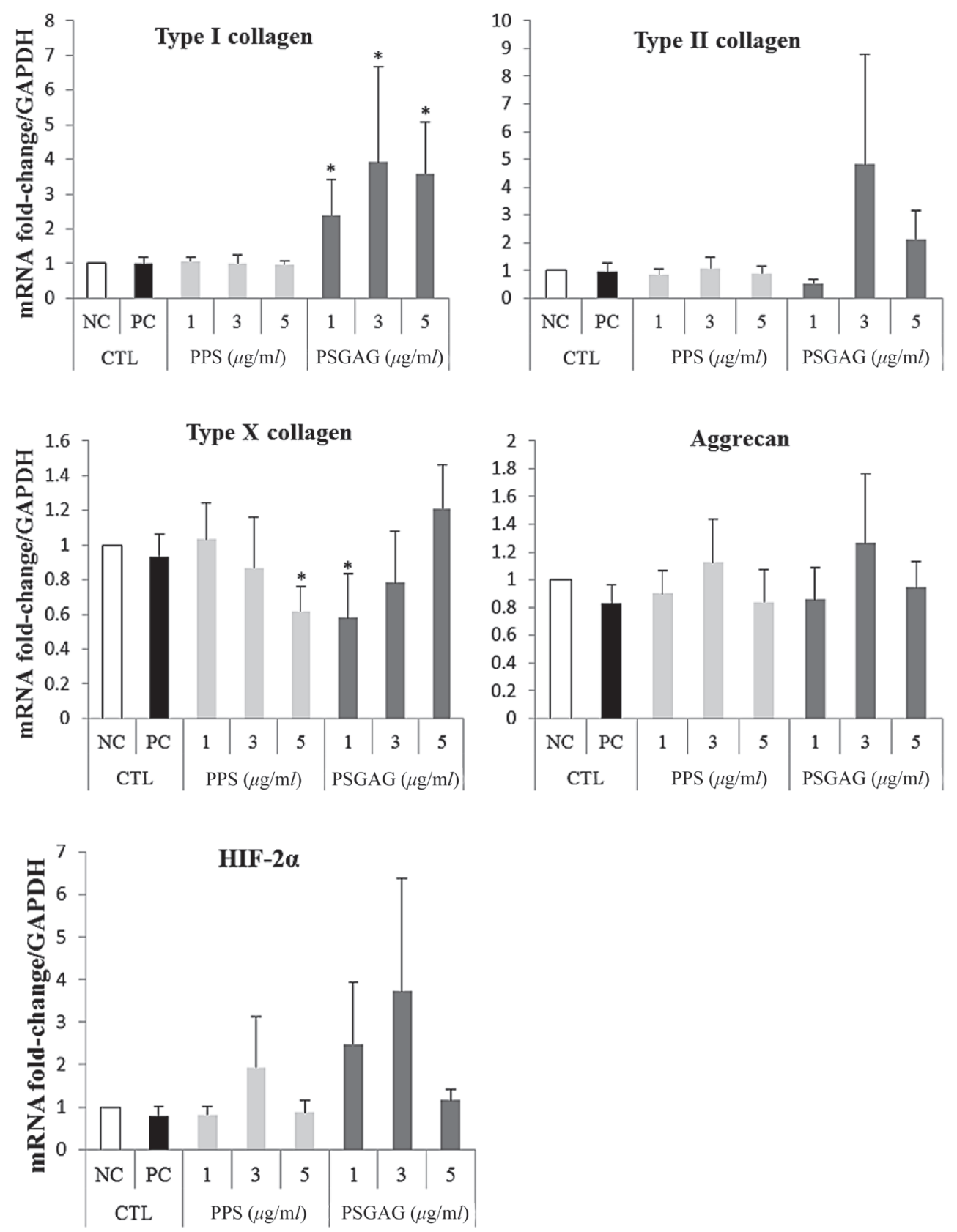

Fig. 1. Effects of pentosan polysulfate (PPS) sodium and polysulfated glycosaminoglycan (PSGAG) on chondrogenesis of canine bone marrowderived mesenchymal stem cells (cBMSC) in alginate beads. Chondrogenic differentiation medium (CDM) (A10071-01) was supplemented with either PPS or PSGAG at 0 (PC), 1, 3 and $5 \mu \mathrm{g} / \mathrm{m} l$. Chondrogenic phenotype gene expression was quantified by real-time PCR targeting chondrocyte-specific genes; type II collagen and aggrecan, dedifferentiation and hypertrophy genes; type I collagen and X collagen, respectively, and hypoxia inducible factor- $2 \alpha$ subunit $(H I F-2 \alpha)$. Glyceraldehyde-3-phosphate dehydrogenase (GAPDH) was used as reference gene. Control (CTL) groups were; negative control (NC); 10\% DMEM, and positive control (PC); CDM. Data represent the mean $\pm 95 \%$ confidence interval $(95 \% \mathrm{CI})$ of 6 independent experiments $(\mathrm{n}=6)$. Significant difference was defined as $* P<0.05$.

MSC encapsulated in alginate beads cultures [28]. However, there are no studies that have evaluated Sox-9 expression in cBMSC cultured in alginate beads therefore our finding could suggest a difference in response of MSC sourced from different species to alginate. We have further verified in this study that when cBMSC are cultured in a monolayer system at a high seeding density, Sox-9 is induced and restored. In addition, we have verified these suppressive effects of two different grades of alginate on Sox-9 mRNA in canine articular chondrocytes which is not dependent on cell seeding density (unpublished data). Therefore, albeit Sox-9 being an established regulator and enhancer of type II collagen, the induction of the gene in the absence of Sox-9 mRNA strongly suggests that it may not be the only key regulator of type II collagen promoter activity although could still be involved at very low physiological levels in maintaining chondrocytes phenotype and this is in agreement with observations made elsewhere [2, 41]. 

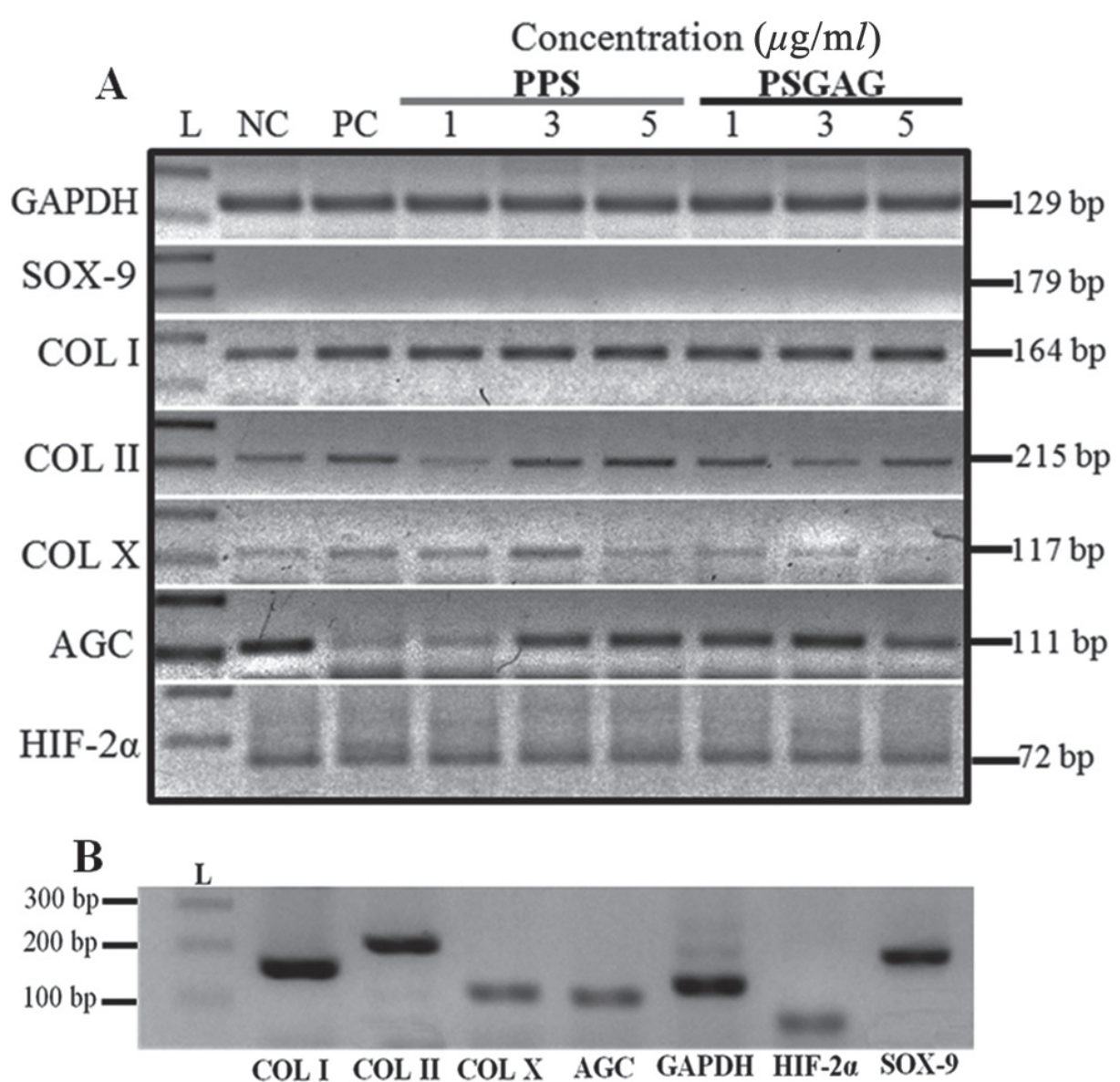

Fig. 2. Alginate beads culture suppresses Sox-9 mRNA expression in canine bone marrow-derived mesenchymal stem cells (cBMSC). (A) Alginate encapsulated cBMSC presented in Fig. 1 were further evaluated for Sox-9 mRNA expression reverse transcriptase-PCR (RT-PCR) ethidium bromide gel electrophoresis since initial assaying by qPCR showed no detectable Sox-9 mRNA signal albeit cells expressing type II collagen and aggrecan. RT-PCR verified that Sox-9 mRNA was suppressed in cBMSC cultured in alginate beads whereas type I, II and X collagen, aggrecan and Hypoxia Inducible Factor-2 $\alpha$ subunit (HIF-2 $\alpha$ ) mRNA bands were all detectable. Glyceraldehyde-3-phosphate dehydrogenase (GAPDH was used as reference gene. L; $100 \mathrm{bp}$ DNA ladder. Gels shown are from one representative experiment of six independent experiments ( $\mathrm{n}=6$ ), (B) shows that contrary to alginate beads culture, monolayer cBMSC cultured in CDM express Sox-9 mRNA and exhibit a chondrogenic phenotype.

The expression of type II collagen and aggrecan in alginate encapsulated cBMSC cultured in only 10\% DMEM demonstrates independent chondrogenesis in the absence of chondroinductive factor previously reported to be necessary [8, 23, 31, 32]. However, similar to findings by others $[5,6,15,29,31,32,39]$, chondrogenic differentiated cBMSC in alginate beads also concomitantly expressed type I and X collagen in the presence or absence of both PPS and PSGAG. These findings taken together indicate that the use of MSC as a source of alginate differentiated chondrocytes population for cartilage tissue regeneration still requires a lot of effort in establishing suitable in vitro culture conditions that can best promote chondrogenesis without the cells progressively undergoing hypertrophy or dedifferentiation. While PPS showed no significant effect on type I collagen mRNA in alginate culture it has been shown to inhibit type I collagen gene expression in human MPC cultured in MMC system [15]. Our study further shows that $H I F-2 \alpha$ mRNA is extensively expressed in cBMSC cultured in alginate beads. While both PPS and PSGAG had no significant effect on HIF-2 $\alpha$ mRNA expression in alginate culture, it was upregulated by both drugs at a concentration $3 \mu \mathrm{g} / \mathrm{m} l$ which also correlated with a non-significant increase in type II collagen and aggrecan. Increased expression of HIF-2 $\alpha$ mRNA has been reported to improve chondrogenic differentiation of human BMSC and stem cells derived from the infrapatellar fat pad under hypoxic conditions $[1,21]$. HIF-2 $\alpha$ has been shown to be essential for hypoxic induction of the human articular chondrocyte phenotype at both the gene and protein level [25] by acting as a promoter of both Sox-9-dependent and independent factors important for key cartilage matrix synthesis [24]. Therefore, the results of our study suggests that $H I F-2 \alpha$ may play a role in the regulation of type II collagen and aggrecan during chondrogenesis in what appears to be Sox-9-independent.

In contrast to alginate culture, in MMC PPS significantly enhanced cBMSC chondrogenic condensation and PG deposition compared to NC, PC and PSGAG. This observation confirms the results of a previous study which demonstrated that PPS promotes proliferation and chondrogenic differentiation of human MPC [15]. In contrast, PSGAG inhibited chondrogenic pelleting and PG deposition in a dose-dependent pattern and resulted in chondrogenic pellets that were sparsely arranged with a fibroblastic-like phenotype that poorly stained with Alcian blue indicating a decreased PG deposition. This effect of PSGAG on chondrogenic 

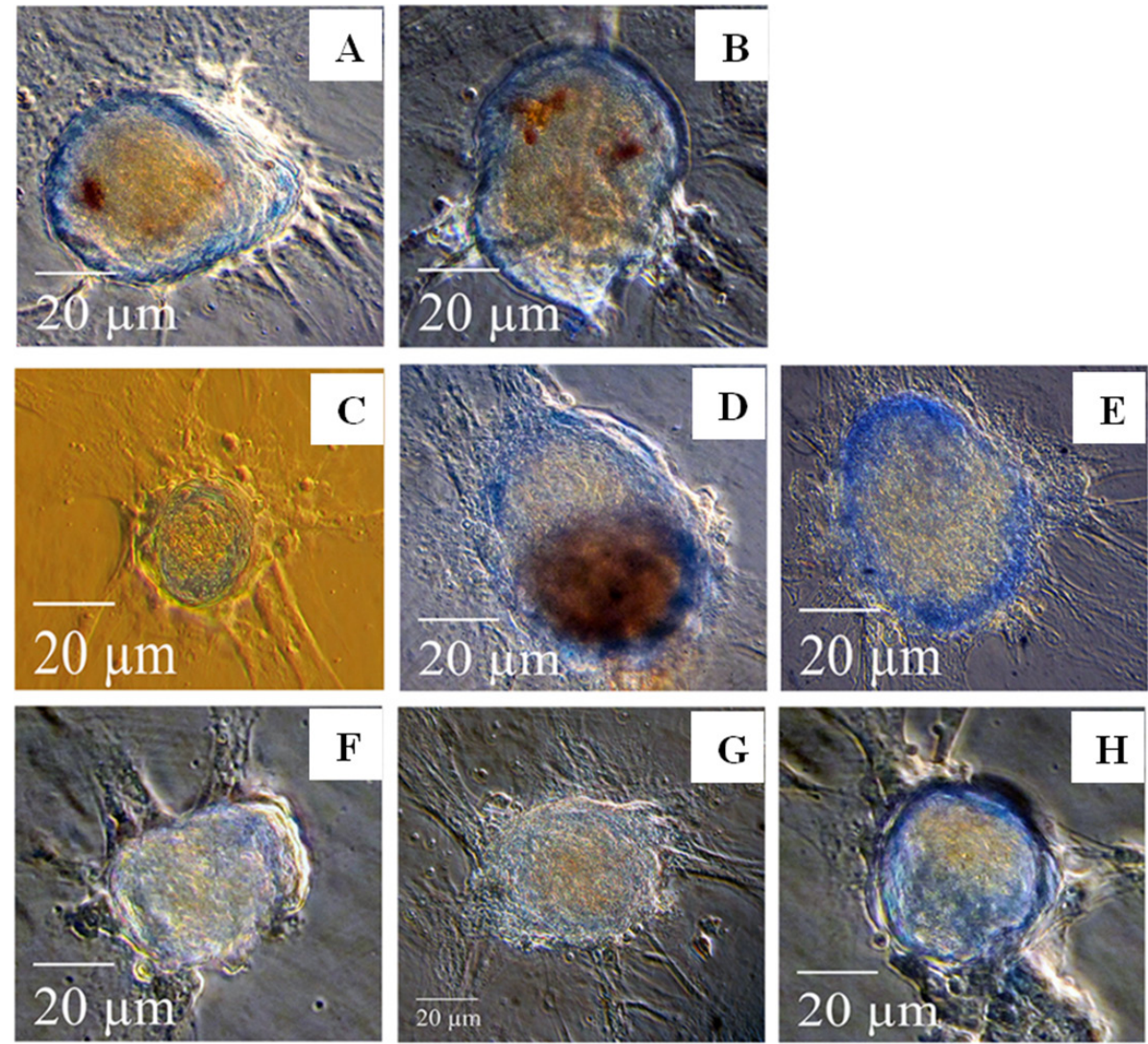

Fig. 3. Effects of pentosan polysulfate (PPS) sodium and polysulfated glycosaminoglycan (PSGAG) on chondrogenesis of canine bone marrowderived mesenchymal stem cells (cBMSC) in micromass culture maintained for 12-days. Photomicrographs represent the treatment conditions as; (A) 10\% DMEM -negative control (NC), (B) chondrogenic differentiation medium (CDM) -positive control (PC), (C) $1.0 \mu \mathrm{g} / \mathrm{m} l \mathrm{PPS},(\mathrm{D})$ $3.0 \mu \mathrm{g} / \mathrm{m} l$ PPS, (E) $5.0 \mu \mathrm{g} / \mathrm{m} l$ PPS, (F) $1.0 \mu \mathrm{g} / \mathrm{m} l$ PSGAG, (G) $3.0 \mu \mathrm{g} / \mathrm{m} l$ PSGAG and (H) $5.0 \mu \mathrm{g} / \mathrm{m} l$ PSGAG. The supplementation of PPS and PSGAG to CDM tended to enhance cell aggregation and PG deposition at 12-days compared to NC and PC although the difference between PPS and PSGAG was not remarkable. PG deposition was evaluated by Alcian blue stain. Results are from one representative of six independent experiments $(\mathrm{n}=6)$. Scale bars: $20 \mu \mathrm{m}$.

differentiation of cBMSC in MMC system could be associated with its previously reported inhibitory effect on growth of cell cultures [16]. Therefore, PSGAG may ultimately fail to stimulate MSC replication, a prerequisite for cartilage tissue regeneration especially in long-term pellet culture systems. The observed fibroblastic-like pellet phenotype in MMC could also be associated with the observed significant upregulation of type I collagen mRNA at all PSGAG concentrations in alginate culture. These findings taken together clearly demonstrate that the response of MSC is dependent on the culture system and varies significantly between alginate and MMC. Of note was that PG deposition was higher 12-days MMC in NC than in 20-days culture. Notably, in 20-days culture, cells in NC proliferated mostly as monolayer fibroblastic-like cells with limited chondrogenic pelleting and PG deposition as indicated by poor Alcian blue stain. Overall, while cells in the PC continued to form compact chondrogenic pellets and exhibited a positive homogenous Alcian blue stain indicating PG deposition, some pellets showed reduced PG deposition albeit chondrogenic pelleting when compared to 12-days MMC. The reduced chondrogenic potential of cBMSC cultured for 20-days in medium without chondroinductive factors $(\mathrm{NC})$ indicates that such cultures generally progress into a mixed fibroblastic-like phenotype synthesizing type I and X collagen whereas the reduced PG deposition in some chondrogenic pellets cultured in CDM (PC) could indicate formation of mixed phenotype pellets in long-term adherent MMC conditions.

In conclusion, we demonstrate that while PPS and PSGAG share a similar chemical structure, their effects on chondrogenesis of MSC varies significantly. The study demonstrates that the response of MSC to chondroinductive factors is culture systemdependent and varies significantly between alginate and MMC. While PPS had no significant effect on chondrogenesis of cBMSC in alginate beads, in MMC it significantly promoted chondrogenesis as indicated by enhanced chondrogenic condensation and PG deposition. In contrast, PSGAG inhibited chondrogenic condensation and PG deposition resulting in sparsely arranged fibroblasticlike pellets with decreased PG deposition and therefore its use in cartilage tissue engineering may be limited. While the present study confirms PPS as suitable alternative chondroinductive factor that may be used in cartilage tissue regeneration and repair of OA joints, caution must be exercised when extrapolating results from one culture system to another as a major variation of MSC response to different culture systems exists. 

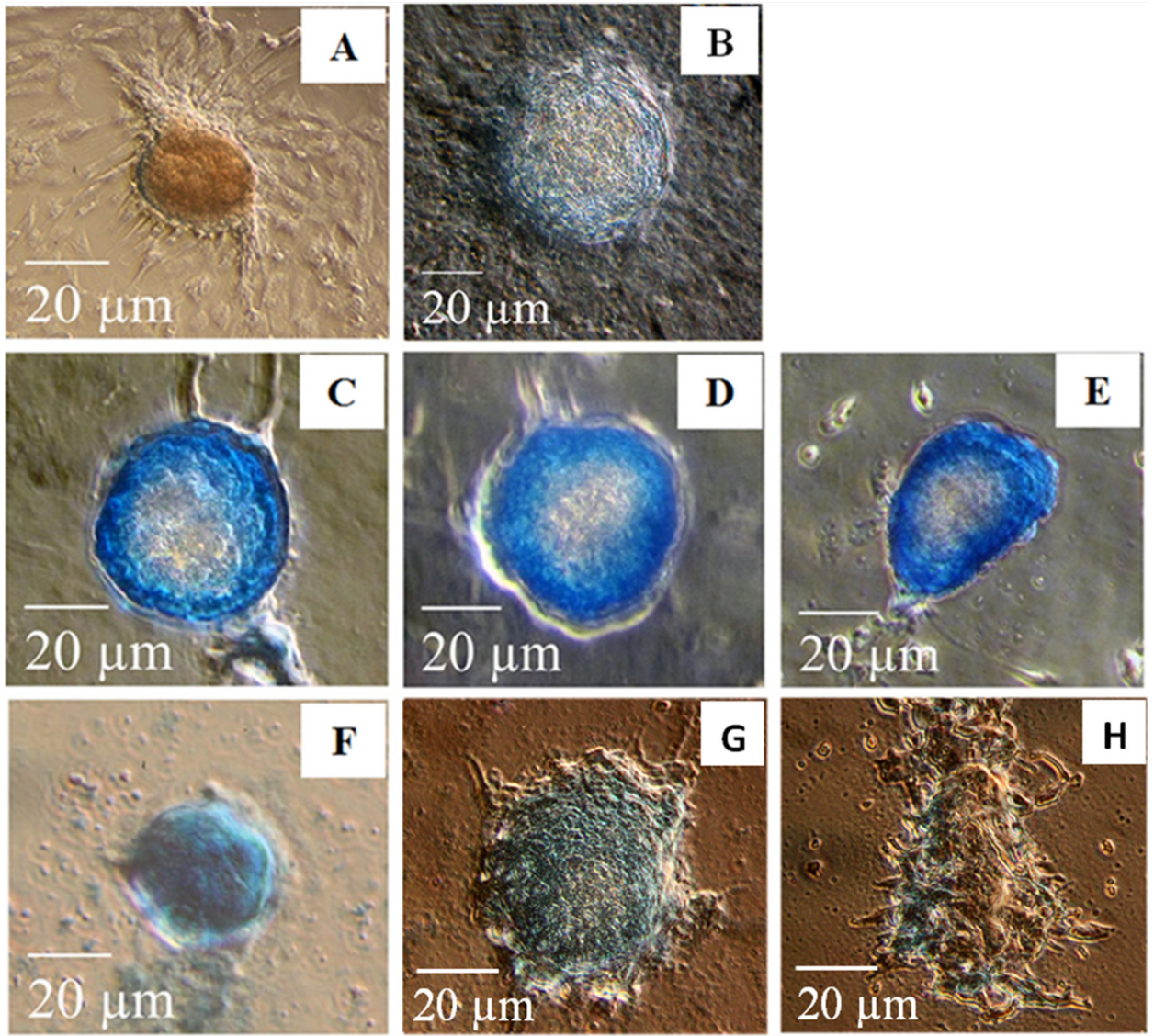

Fig. 4. Effects of pentosan polysulfate (PPS) sodium and polysulfated glycosaminoglycan (PSGAG) on chondrogenesis of canine bone marrowderived mesenchymal stem cells (cBMSC) in micromass culture maintained for 20-days. Photomicrographs represent the treatment conditions as; (A) 10\% DMEM -negative control (NC), (B) chondrogenic differentiation medium (CDM) -positive control (PC), (C) $1.0 \mu \mathrm{g} / \mathrm{m} l \mathrm{PPS},(\mathrm{D}) 3.0$ $\mu \mathrm{g} / \mathrm{m} l$ PPS, (E) $5.0 \mu \mathrm{g} / \mathrm{m} l$ PPS, (F) $1.0 \mu \mathrm{g} / \mathrm{m} l$ PSGAG, (G) $3.0 \mu \mathrm{g} / \mathrm{m} l$ PSGAG and (H) $5.0 \mu \mathrm{g} / \mathrm{m} l$ PSGAG. In 20-days culture, the supplementation of PPS to CDM significantly enhanced chondrogenic pelleting and PG deposition at all concentrations compared to NC, PC and PSGAG as indicated by intense and homogenous Alcian blue staining of pellets. In contrast, PSGAG inhibited chondrogenic pellet formation in a dosedependent pattern leading to formation of fibroblastic-like pellets that stained poorly with Alcian blue, an indication of reduced PG deposition. Results are from one representative of six independent experiments (n=6). Scale bars: $20 \mu \mathrm{m}$.

\section{REFERENCES}

1. Adesida, A. B., Mulet-Sierra, A. and Jomha, N. M. 2012. Hypoxia mediated isolation and expansion enhances the chondrogenic capacity of bone marrow mesenchymal stromal cells. Stem Cell Res. Ther. 3: 9. [Medline] [CrossRef]

2. Aigner, T., Gebhard, P. M., Schmid, E., Bau, B., Harley, V. and Pöschl, E. 2003. SOX9 expression does not correlate with type II collagen expression in adult articular chondrocytes. Matrix Biol. 22: 363-372. [Medline] [CrossRef]

3. Barbero, A., Grogan, S., Schäfer, D., Heberer, M., Mainil-Varlet, P. and Martin, I. 2004. Age related changes in human articular chondrocyte yield, proliferation and post-expansion chondrogenic capacity. Osteoarthritis Cartilage 12: 476-484. [Medline] [CrossRef]

4. Bonaventure, J., Kadhom, N., Cohen-Solal, L., Ng, K. H., Bourguignon, J., Lasselin, C. and Freisinger, P. 1994. Reexpression of cartilage-specific genes by dedifferentiated human articular chondrocytes cultured in alginate beads. Exp. Cell Res. 212: 97-104. [Medline] [CrossRef]

5. Bosnakovski, D., Mizuno, M., Kim, G., Takagi, S., Okumur, M. and Fujinag, T. 2006. Gene expression profile of bovine bone marrow mesenchymal stem cell during spontaneous chondrogenic differentiation in pellet culture system. Jpn. J. Vet. Res. 53: 127-139. [Medline]

6. Bosnakovski, D., Mizuno, M., Kim, G., Ishiguro, T., Okumura, M., Iwanaga, T., Kadosawa, T. and Fujinaga, T. 2004. Chondrogenic differentiation of bovine bone marrow mesenchymal stem cells in pellet cultural system. Exp. Hematol. 32: 502-509. [Medline] [CrossRef]

7. Budsberg, S. C., Bergh, M. S., Reynolds, L. R. and Streppa, H. K. 2007. Evaluation of pentosan polysulfate sodium in the postoperative recovery from cranial cruciate injury in dogs: a randomized, placebo-controlled clinical trial. Vet. Surg. 36: 234-244. [Medline] [CrossRef]

8. Coleman, R. M., Case, N. D. and Guldberg, R. E. 2007. Hydrogel effects on bone marrow stromal cell response to chondrogenic growth factors. Biomaterials 28: 2077-2086. [Medline] [CrossRef]

9. Collier, S. and Ghosh, P. 1989. Evaluation of the effect of antiarthritic drugs on the secretion of proteoglycans by lapine chondrocytes using a novel assay procedure. Ann. Rheum. Dis. 48: 372-381. [Medline] [CrossRef]

10. Costeseque, R., Emonds-Alt, X., Breliere, J. C. and Roncucci, R. 1986. Polysulphated polysaccharides: an in vitro study of their effects on proteoglycan biosynthesis by articular chondrocytes. Arch. Int. Pharmacodyn. Ther. 282: 196-208. [Medline]

11. Darling, E. M. and Athanasiou, K. A. 2005. Rapid phenotypic changes in passaged articular chondrocyte subpopulations. J. Orthop. Res. 23: 425-432. [Medline] [CrossRef]

12. Freshney, I. R. 2010. Culture of animal cells; A manual of basic technique and specialized applications, 6th ed., Wiley-Blackwell, New Jersey. 
13. Fujiki, M., Shineha, J., Yamanokuchi, K., Misumi, K. and Sakamoto, H. 2007. Effects of treatment with polysulfated glycosaminoglycan on serum cartilage oligomeric matrix protein and C-reactive protein concentrations, serum matrix metalloproteinase-2 and -9 activities, and lameness in dogs with osteoarthritis. Am. J. Vet. Res. 68: 827-833. [Medline] [CrossRef]

14. Ghosh, P. 1999. The pathobiology of osteoarthritis and the rationale for the use of pentosan polysulfate for its treatment. Semin. Arthritis Rheum. 28: 211-267. [Medline] [CrossRef]

15. Ghosh, P., Wu, J., Shimmon, S., Zannettino, A. C., Gronthos, S. and Itescu, S. 2010. Pentosan polysulfate promotes proliferation and chondrogenic differentiation of adult human bone marrow-derived mesenchymal precursor cells. Arthritis Res. Ther. 12: R28. [Medline] [CrossRef]

16. Glade, M. J. 1990. Polysulfated glycosaminoglycan accelerates net synthesis of collagen and glycosaminoglycans by arthritic equine cartilage tissues and chondrocytes. Am. J. Vet. Res. 51: 779-785. [Medline]

17. Hamada, T., Sakai, T., Hiraiwa, H., Nakashima, M., Ono, Y., Mitsuyama, H. and Ishiguro, N. 2013. Surface markers and gene expression to characterize the differentiation of monolayer expanded human articular chondrocytes. Nagoya J. Med. Sci. 75: 101-111. [Medline]

18. Hellio Le Graverand-Gastineau, M. P. 2009. OA clinical trials: current targets and trials for OA. Choosing molecular targets: what have we learned and where we are headed? Osteoarthritis Cartilage 17: 1393-1401. [Medline] [CrossRef]

19. Ikeda, T., Kawaguchi, H., Kamekura, S., Ogata, N., Mori, Y., Nakamura, K., Ikegawa, S. and Chung, U. I. 2005. Distinct roles of Sox5, Sox6, and Sox9 in different stages of chondrogenic differentiation. J. Bone Miner. Metab. 23: 337-340. [Medline] [CrossRef]

20. Innes, J. F., Barr, A. R. and Sharif, M. 2000. Efficacy of oral calcium pentosan polysulphate for the treatment of osteoarthritis of the canine stifle joint secondary to cranial cruciate ligament deficiency. Vet. Rec. 146: 433-437. [Medline] [CrossRef]

21. Khan, W. S., Adesida, A. B. and Hardingham, T. E. 2007. Hypoxic conditions increase hypoxia-inducible transcription factor 2alpha and enhance chondrogenesis in stem cells from the infrapatellar fat pad of osteoarthritis patients. Arthritis Res. Ther. 9: R55. [Medline] [CrossRef]

22. Krasnokutsky, S., Attur, M., Palmer, G., Samuels, J. and Abramson, S. B. 2008. Current concepts in the pathogenesis of osteoarthritis. Osteoarthritis Cartilage 16 Suppl 3: S1-S3. [Medline] [CrossRef]

23. Kyriacos, A. A., Darling, E. M., DuRaine, G. D., Hu, J. C. and Reddi, A. H. 2013 Articular cartilage. Taylor \& Francis Group, Florida.

24. Lafont, J. E., Talma, S. and Murphy, C. L. 2007. Hypoxia-inducible factor 2alpha is essential for hypoxic induction of the human articular chondrocyte phenotype. Arthritis Rheum. 56: 3297-3306. [Medline] [CrossRef]

25. Lafont, J. E., Talma, S., Hopfgarten, C. and Murphy, C. L. 2008. Hypoxia promotes the differentiated human articular chondrocyte phenotype through SOX9-dependent and -independent pathways. J. Biol. Chem. 283: 4778-4786. [Medline] [CrossRef]

26. Lemare, F., Steimberg, N., Le Griel, C., Demignot, S. and Adolphe, M. 1998. Dedifferentiated chondrocytes cultured in alginate beads: restoration of the differentiated phenotype and of the metabolic responses to interleukin-lbeta. J. Cell. Physiol. 176: 303-313. [Medline] [CrossRef]

27. Leung, V. Y. L., Gao, B., Leung, K. K. H., Melhado, I. G., Wynn, S. L., Au, T. Y. K., Dung, N. W. F., Lau, J. Y. B., Mak, A. C. Y., Chan, D. and Cheah, K. S. E. 2011. SOX9 governs differentiation stage-specific gene expression in growth plate chondrocytes via direct concomitant transactivation and repression. PLoS Genet. 7: e1002356. [Medline] [CrossRef]

28. Majumdar, M. K., Wang, E. and Morris, E. A. 2001. BMP-2 and BMP-9 promotes chondrogenic differentiation of human multipotential mesenchymal cells and overcomes the inhibitory effect of IL-1. J. Cell. Physiol. 189: 275-284. [Medline] [CrossRef]

29. Mauck, R. L., Yuan, X. and Tuan, R. S. 2006. Chondrogenic differentiation and functional maturation of bovine mesenchymal stem cells in longterm agarose culture. Osteoarthritis Cartilage 14: 179-189. [Medline] [CrossRef]

30. Munteanu, S. E., Ilic, M. Z. and Handley, C. J. 2000. Calcium pentosan polysulfate inhibits the catabolism of aggrecan in articular cartilage explant cultures. Arthritis Rheum. 43: 2211-2218. [Medline] [CrossRef]

31. Mwale, F., Stachura, D., Roughley, P. and Antoniou, J. 2006. Limitations of using aggrecan and type X collagen as markers of chondrogenesis in mesenchymal stem cell differentiation. J. Orthop. Res. 24: 1791-1798. [Medline] [CrossRef]

32. Nöth, U., Rackwitz, L., Heymer, A., Weber, M., Baumann, B., Steinert, A., Schütze, N., Jakob, F. and Eulert, J. 2007. Chondrogenic differentiation of human mesenchymal stem cells in collagen type I hydrogels. J. Biomed. Mater. Res. A 83: 626-635. [Medline] [CrossRef]

33. Qvist, P., Bay-Jensen, A. C., Christiansen, C., Dam, E. B., Pastoureau, P. and Karsdal, M. A. 2008. The disease modifying osteoarthritis drug (DMOAD): Is it in the horizon? Pharmacol. Res. 58: 1-7. [Medline] [CrossRef]

34. Rogachefsky, R. A., Dean, D. D., Howell, D. S. and Altman, R. D. 1993. Treatment of canine osteoarthritis with insulin-like growth factor-1 (IGF-1) and sodium pentosan polysulfate. Osteoarthritis Cartilage 1: 105-114. [Medline] [CrossRef]

35. Sevalla, K., Todhunter, R. J., Vernier-Singer, M. and Budsberg, S. C. 2000. Effect of polysulfated glycosaminoglycan on DNA content and proteoglycan metabolism in normal and osteoarthritic canine articular cartilage explants. Vet. Surg. 29: 407-414. [Medline] [CrossRef]

36. Shen, M., Yoshida, E., Yan, W., Kawamoto, T., Suardita, K., Koyano, Y., Fujimoto, K., Noshiro, M. and Kato, Y. 2002. Basic helix-loop-helix protein DEC1 promotes chondrocyte differentiation at the early and terminal stages. J. Biol. Chem. 277: 50112-50120. [Medline] [CrossRef]

37. Singh, M., Pierpoint, M., Mikos, A. G. and Kasper, F. K. 2011. Chondrogenic differentiation of neonatal human dermal fibroblasts encapsulated in alginate beads with hydrostatic compression under hypoxic conditions in the presence of bone morphogenetic protein-2. J. Biomed. Mater. Res. A 98: 412-424. [Medline] [CrossRef]

38. Soleimani, M. and Nadri, S. 2009. A protocol for isolation and culture of mesenchymal stem cells from mouse bone marrow. Nat. Protoc. 4: 102-106. [Medline] [CrossRef]

39. Studer, D., Millan, C., Öztürk, E., Maniura-Weber, K. and Zenobi-Wong, M. 2012. Molecular and biophysical mechanisms regulating hypertrophic differentiation in chondrocytes and mesenchymal stem cells. Eur. Cell. Mater. 24: 118-135, discussion 135. [Medline] [CrossRef]

40. Sunaga, T., Oh, N., Hosoya, K., Takagi, S. and Okumura, M. 2012. Inhibitory effects of pentosan polysulfate sodium on MAP-kinase pathway and NF-kB nuclear translocation in canine chondrocytes in vitro. J. Vet. Med. Sci. 74: 707-711. [Medline] [CrossRef]

41. Takahashi, I., Nuckolls, G. H., Takahashi, K., Tanaka, O., Semba, I., Dashner, R., Shum, L. and Slavkin, H. C. 1998. Compressive force promotes sox9, type II collagen and aggrecan and inhibits IL-1beta expression resulting in chondrogenesis in mouse embryonic limb bud mesenchymal cells J. Cell Sci. 111: 2067-2076. [Medline]

42. Tallheden, T., Bengtsson, C., Brantsing, C., Sjögren-Jansson, E., Carlsson, L., Peterson, L., Brittberg, M. and Lindahl, A. 2005. Proliferation and differentiation potential of chondrocytes from osteoarthritic patients. Arthritis Res. Ther. 7: R560-R568. [Medline] [CrossRef]

43. Todhunter, R. J. and Lust, G. 1994. Polysulfated glycosaminoglycan in the treatment of osteoarthritis. J. Am. Vet. Med. Assoc. 204: 1245-1251. [Medline]

44. Vaughan-Scott, T. and Taylor, J. H. 1997. The pathophysiology and medical management of canine osteoarthritis. J. S. Afr. Vet. Assoc. 68: 21-25. [Medline] [CrossRef] 